\title{
Complete genome sequence of Paenibacillus konkukensis sp. nov. SK3146 as a potential probiotic strain
}

\author{
Hae-In Jung ${ }^{1 \#}$, Sungkwon Park ${ }^{2 \#}$, Kai-Min Niư ${ }^{3}$, Sang-Won Lee ${ }^{4}$, \\ Damini Kothari ${ }^{1}$, Kwon Jung Yi ${ }^{1}$ and Soo-Ki Kim ${ }^{1 *}$ \\ ${ }^{1}$ Department of Animal Sciences and Technology, Konkuk University, Seoul 05029, Korea \\ ${ }^{2}$ Department of Food Science and Biotechnology, Sejong University, Seoul 05005, Korea \\ ${ }^{3}$ Institute of Biological Resources, Jiangxi Academy of Sciences, Nanchang 330096, China \\ ${ }^{4}$ College of Veterinary Medicine, Konkuk University, Seoul 05029, Korea
}

Received: Mar 5, 2021

Revised: Mar 15, 2021

Accepted: Mar 15, 2021

\#These authors contributed equally to this work.

${ }^{*}$ Corresponding author

Soo-Ki Kim

Department of Animal Sciences and

Technology, Konkuk University, Seoul

05029, Korea.

Tel: +82-2-450-3728

E-mail: sookikim@konkuk.ac.kr

Copyright $\odot 2021$ Korean Society of Animal Sciences and Technology.

This is an Open Access article distributed under the terms of the Creative Commons Attribution

Non-Commercial License (http:// creativecommons.org/licenses/by$\mathrm{nc} / 4.0 /$ ) which permits unrestricted non-commercial use, distribution, and reproduction in any medium, provided the original work is properly cited.

ORCID

Hae-In Jung

https://orcid.org/0000-0003-2643-6681 Sungkwon Park

https://orcid.org/0000-0002-7684-9719 Kai-Min Niu

https://orcid.org/0000-0002-9756-3517

Sang-Won Lee

https://orcid.org/0000-0003-1956-7245

Damini Kothari

https://orcid.org/0000-0003-3627-2377

Kwon Jung Yi

https://orcid.org/0000-0001-6002-0378

\begin{abstract}
Paenibacillus konkukensis sp. nov., SK3146 is a novel strain isolated from a pig feed. Here, we present complete genome sequence of SK3146. The genome consists of a single circular genome measuring 7,968,964 bp in size with an average guanine + cytosine $(\mathrm{G}+\mathrm{C})$ content of $53.4 \%$. Genomic annotation revealed that the strain encodes 151 proteins related to hydrolases (EC3), which was higher than those in Bacillus subtilis and Escherichia coli. Diverse kinds of hydrolases including galactosidase, glucosidase, cellulase, lipase, xylanase, and protease were found in the genome of SK3146, coupled with one bacteriocin encoding gene. The complete genome sequence of $P$. konkukensis SK3146 indicates the immense probiotic potential of the strain with nutrient digestibility and antimicrobial activity functions.
\end{abstract}

Keywords: Paenibacillus, Complete genome sequence, Exoenzyme, Feed additive

The well-being and health status of economic animals are more deteriorative due to the intensive farming practices. Feed-additive probiotics including lactic acid bacteria and Bacillus spp. are commonly used to modulate host health and improve performance in pig production $[1,2]$. Typically, a culturedependent isolation coupled with in-vitro characterization methods have been used to establish probiotic potential of the strains. Due to rapid development of next generation sequencing in the past decade, scientists are now exploring whole genome sequencing to identify and functionally characterize novel probiotic strains. A number of potential probiotic strains have been identified based on their putative functional genes from their whole genome [3].

Paenibacillus spp. are well known as growth promoters for crops, but there are very limited studies exploring their probiotic potentials, despite the fact that they can produce diverse kinds of active substances namely antimicrobial peptides (bacteriocin and lipopeptide), volatile organic compounds, and digestive enzymes (amylase, cellulase, lipase, protease, etc), among many others [4].

Previously, we have isolated a novel Paenibacillus strain SK3146 $6^{\mathrm{T}}\left(=\right.$ KACC $18876^{\mathrm{T}}=\mathrm{LMG} 29568^{\mathrm{T}}$ ) from a pig feed, which was taxonomically assigned as Paenibacillus konkukensis sp. nov. [5]. In this study, 
Soo-Ki Kim

https://orcid.org/0000-0003-3499-3330

Competing interests

No potential conflict of interest relevant to

this article was reported.

Funding sources

This research was supported by a grant from Agricultural Science and Technology Development Program (Project No. PJ010906), Rural Development Administration, Korea.

Acknowledgements

Not applicable.

Availability of data and material Upon reasonable request, the datasets of this study can be available from the corresponding author.

Authors' contributions

Conceptualization: Jung HI, Park S, Lee SW, Kim SK.

Data curation: Jung HI.

Formal analysis: Jung HI, Niu KM.

Methodology: Jung $\mathrm{HI}$.

Validation: Jung HI, Lee SW.

Investigation: Jung HI, Yi KJ.

Writing - original draft: Jung HI, Lee SW.

Writing - review \& editing: Park S, Niu KM, Lee SW, Kothari D, Kim SK.

Ethics approval and consent to participate This article does not require IRB/IACUC approval because there are no human and animal participants. we provide a detailed description of the complete genome sequence of SK3146 and analyzed its putative functional genes related to digestive enzymes and bacteriocin which could be beneficial attributes as a functional feed additive.

SK3146 was cultured in Luria-Bertani broth for two days at $37^{\circ} \mathrm{C}$ under shaking conditions (100 rpm). Genomic DNA of SK3146 was extracted using the Wizard Genomic DNA Purification Kit (Promega, Madison, WI, USA) according to the manufacturer's instructions. The genome of SK3146 was completely sequenced using the PacBio ${ }^{\circledR}$ RS II system by Macrogen (Seoul, Korea). The PacBio RS II system libraries were prepared using the SMRTbell template prep kit v 1.0. In total, 142,242 bp PacBio subreads with 1,041,901,553 bp were generated using the PacBio ${ }^{\circledR}$ RS II system, and their mean length and N50 value were 7,324 and 10,697 bp, respectively. The sequencing reads were de novo assembled using the HGAP analysis with default options. The assembly was completed with the PacBio RS II system. Annotation of coding DNA sequence (CDS) and functional genes were analyzed by the Prokka v1.10. The general features of the SK3146 were analyzed based on its complete genome sequence using the Geneious 8.1.9 software (Biomatters, Auckland, New Zealand) [6]. The predicted CDS were classified depending on the clusters of orthologous genes (COG), followed by the construction of a circular genome map and analysis of protein function by a web server: Bacterial Annotation System (https://www.basys.ca/) [7].

The circular genome visualization and general features of SK3146 genome are presented in Fig. 1 and summarized in Table 1, respectively. The complete genome of the strain consists of a single circular chromosome measuring 7,968,964 bp in size and 53.4\% in guanine + cytosine $(\mathrm{G}+\mathrm{C})$ content. A total of 6,988 genes were predicted in the genome including 6,842 CDS, 37 ribosomal RNAs (rRNA), 108 transfer RNAs (tRNA), and 1 transfer-messenger RNA (tmRNA) loci. In addition, 10 clustered regularly interspaced short palindromic repeats (CRISPR) elements, three
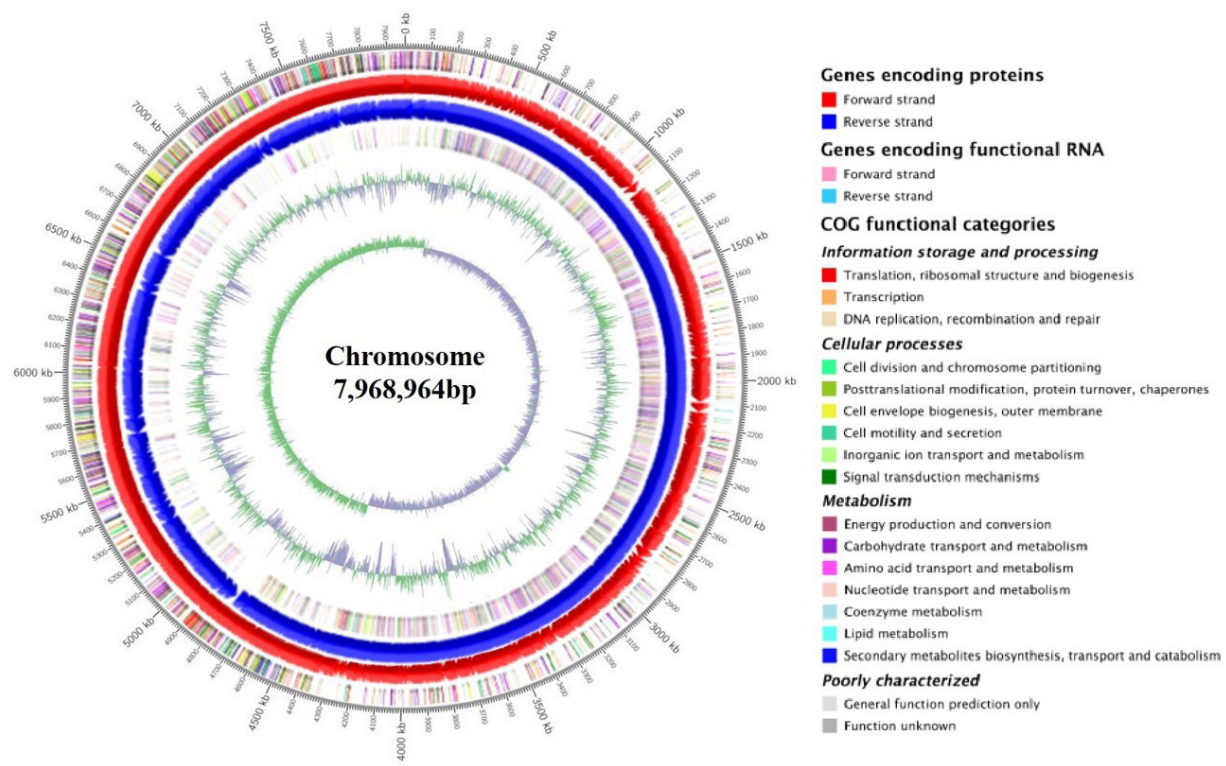

Fig. 1. Circular genome map of Paenibacillus konkukensis sp. nov. SK3146. Marked characteristics are shown from outside to the center: COG annotation gene distribution on the forward strand; CDS on the forward DNA strand (red); CDS on the reverse DNA strand (blue); COG annotation gene distribution on the reverse strand; GC content, and GC skew. The lines in each concentric circle indicate the position of the represented feature; the color key is presented on the right side of the map. COG, clusters of orthologous genes; CDS, coding DNA sequence; G, guanine; C, cytosine. 
Table 1. General features of Paenibacillus konkukensis SK3146 genome

\begin{tabular}{lr}
\hline \multicolumn{1}{c}{ Attributes } & Value \\
\hline Length of sequence (bp) & $7,968,964$ \\
G+C content (\%) & 53.4 \\
No. of CDS & 6,842 \\
No. of genes & 6,988 \\
$\%$ coding & 86 \\
No. of RNAs & 146 \\
No. of rRNAs & 37 \\
No. of tRNAs & 108 \\
No. of tmRNA & 1 \\
No. of CRISPR regions & 10 \\
No. of prophages & 3 \\
No. of IS & 16
\end{tabular}

G, guanine; C, cytosine; CDS, coding DNA sequence; CRISPR, clustered regularly interspaced short palindromic repeats; elements, IS, insertion sequence.

Table 2. Potential metabolic enzyme determinants in Paenibacillus konkukensis SK3146 genome

\begin{tabular}{|c|c|c|c|}
\hline Exoenzymes & No. of CDS & Product name & EC number ${ }^{1)}$ \\
\hline Arylsulfatase & 38 & Arylsulfatase & 3.1. \\
\hline Arabinofuranohydrolase & 2 & Non-reducing end beta-L-arabinofuranosidase, Arabinoxylan arabinofuranohydrolase precursor & 3.2. \\
\hline Allantoinase & 1 & Allantoinase & 3.5 . \\
\hline Agmatinase & 1 & Agmatinase & 3.5. \\
\hline Arginase & 1 & Arginase & 3.5. \\
\hline$\alpha$-Galactosidase & 5 & Alpha-galactosidase & 3.2. \\
\hline a-Galacturonidase & 1 & Alpha-galacturonidase & 3.2. \\
\hline$\beta$-Glucronidase & 6 & Beta-glucuronidase & 3.2. \\
\hline$\beta$-Glucosidase & 4 & Periplasmic beta-glucosidase precursor, Thermostable beta-glucosidase B, Oligo-1\%2C6-glucosidase & 3.2. \\
\hline$\beta$-Galactosidase & 8 & Beta-galactosidase, Evolved beta-galactosidase subunit alpha & 3.2. \\
\hline$\beta$-Xylosidase & 3 & Beta-xylosidase & 3.2. \\
\hline Cellulase & 7 & Endoglucanase precursor, Endoglucanase Z precursor & 3.2. \\
\hline Chitinase & 1 & Chitinase $\mathrm{A} 1$ precursor & 3.2. \\
\hline D-Aminoacylase & 1 & D-aminoacylase & 3.5 . \\
\hline Galactose & 3 & Galactose/methyl galactoside import ATP-binding protein & 3.6. \\
\hline Glutaminase & 1 & Glutaminase & 3.5 . \\
\hline Glucoamylase & 3 & Glucoamylase precursor & 3.2. \\
\hline Lipase & 1 & Lipase 3 precursor & 3.1. \\
\hline Nitrilase & 1 & Nitrilase & 3.5. \\
\hline Protease & 25 & $\begin{array}{l}\text { Germination protease precursor, Protease } 3 \text { precursor, Putative metalloprotease, Putative zinc } \\
\text { protease, Serine endoprotease, Serine protease Do-like, Serine protease, Rhomboid protease, Car- } \\
\text { boxy-terminal processing protease precursor, putative CtpA-like serine protease, ATP-dependent } \\
\text { protease subunit, putative protease precursor, Sporulation-specific protease, Protease, ATP-depen- } \\
\text { dent protease proteolytic subunit, Lon protease, putative protease, Putative cysteine protease }\end{array}$ & $\begin{array}{l}3.4 . \\
3.2 .\end{array}$ \\
\hline Polygalacturonase & 3 & Polygalacturonase & 3.2 . \\
\hline Thiaminase & 2 & Thiaminase-2 & 3.5 . \\
\hline Urease & 6 & Urease subunit beta, Urease subunit gamma, Urease subunit alpha & 3.5 . \\
\hline Xylanase & 27 & $\begin{array}{l}\text { Endo-1\%2C4-beta-xylanase A precursor, Endo-1\%2C4-beta-xylanase Z precursor, Beta-1\%2C3-xy- } \\
\text { lanase XYL4 precursor }\end{array}$ & 3.2. \\
\hline
\end{tabular}

${ }^{11} \mathrm{EC}$ (Enzyme commission number) is a numerical classification scheme for enzymes, based on the chemical reactions they catalyze. EC 3 enzymes are hydrolases (EC3.1: ester bonds; EC3.2: sugars; EC3.3: ether bonds; EC3.4: Peptide bonds; EC3.5: carbon-nitrogen bonds; EC3.6: acid anhydrides).

CDS, coding DNA sequence; ATP, adenosine triphosphate. 
prophage regions, and 16 insertion sequence (IS) elements were identified in the genome.

Furthermore, we have analyzed the presence of potential enzymes in the genome of SK3146 via protein function annotation with the Kyoto Encyclopedia of Genes and Genomes database, which provides specific substrates, reactions, and enzyme nomenclature [8]. We then categorized the genes encoding potential enzymes of SK3146 according to enzyme code number. The class of hydrolases (EC3) including galactosidase, glucosidase, cellulase, lipase, xylanase, protease, and others in SK3146 are listed in Table 2. EC3 hydrolases including phosphatases, glycosidases, peptidases, nucleosidases, and lipases are widely used in feed additive industry to improve digestibility and bioavailability of nutrients in animal feeds [9]. Besides enzyme encoding genes, one bacteriocin encoding gene was also found on the chromosome of SK3146. The hydrolytic enzymes such as glucanase, cellulase, protease, and chitinase of Paenibacillus have been reported to have anti-fungal activities via destruction of fungal cell wall [10]. Moreover, $\beta$-glucosidase, cellulase, xylanase, and protease have been demonstrated to reduce carbohydrate- and protein-based anti-nutritional factors present in the plant-derived protein sources and consequently improving the nutritional quality of feed [11].

In the present study, the complete genome of P. konkukensis sp. nov., SK3146 isolated from a pig feed has been reported. The genome of SK3146 encodes multiple enzymes that could be applied to improve the digestibility and bioavailability of nutrients of animal feed. A gene encoding bacteriocin was also identified. Thus, the genome mining conducted in this study suggests that stain SK3146 has significant potential as a probiotic for use in feed additive applications. In addition, the genome information of SK3146 widens our understanding on the whole genus of Paenibacillus to explore and develop next generation probiotics. The genome-based protein prediction will be validated by in vitro characterization and in vivo animal study in near future.

\section{GENOMIC SEQUENCE ACCESSION NUMBER}

The complete genome sequence of $P$. konkukensis sp. nov., SK3146 was deposited in the GenBank under the accession number CP027059.

\section{REFERENCES}

1. Barba-Vidal E, Martin-Orue SM, Castillejos L. Practical aspects of the use of probiotics in pig production: a review. Livest Sci. 2019;223:84-96. https://doi.org/10.1016/j.livsci.2019.02.017

2. Chaucheyras-Durand F, Durand H. Probiotics in animal nutrition and health. Benef Microbes. 2010;1:3-9. https://doi.org/10.3920/BM2008.1002

3. Cunningham M, Azcarate-Peril MA, Barnard A, Benoit V, Grimaldi R, Guyonnet D, et al. Shaping the future of probiotics and prebiotics. Trends Microbiol. 2021. https://doi. org/10.1016/j.tim.2021.01.003

4. Grady EN, MacDonald J, Liu L, Richman A, Yuan ZC. Current knowledge and perspectives of Paenibacillus: a review. Microb Cell Fact. 2016;15:203. https://doi.org/10.1186/s12934-0160603-7

5. Im WT, Yi KJ, Lee SS, Moon HI, Jeon CO, Kim DW, et al. Paenibacillus konkukensis sp. nov., isolated from animal feed. Int J Syst Evol Microbiol. 2017;67:2343-8. https://doi.org/10.1099/ ijsem.0.001955

6. Kearse M, Moir R, Wilson A, Stones-Havas S, Cheung M, Sturrock S, et al. Geneious basic: an integrated and extendable desktop software platform for the organization and analysis of sequence data. Bioinformatics. 2012;28:1647-9. https:/doi.org/10.1093/bioinformatics/bts199 
7. Van Domselaar GH, Stothard P, Shrivastava S, Cruz JA, Guo A, Dong X, et al. BASys: a web server for automated bacterial genome annotation. Nucleic Acid Res. 2005;33:W455-9. https:// doi.org/10.1093/nar/gki593

8. McDonald AG, Tipton KF. Fifty-five years of enzyme classification: advances and difficulties. FEBS J. 2014;281:583-92. https://doi.org/10.1111/febs.12530

9. Aureli R, La-Marta J, Grossi AB, Della Pia EA, Esteve-Garcia E, Wulf-Andersen L, et al. A novel glucuronoxylan hydrolase produced by fermentation is safe as feed additive: toxicology and tolerance in broiler chickens. Regul Toxicol Pharmacol. 2018;99:213-24. https://doi. org/10.1016/j.yrtph.2018.09.024

10. Naing KW, Anees M, Kim SJ, Nam Y, Kim YC, Kim KY. Characterization of antifungal activity of Paenibacillus ehimensis KWN38 against soilborne phytopathogenic fungi belonging to various taxonomic groups. Ann Microbiol. 2014;64:55-63. https://doi.org/10.1007/s13213013-0632-y

11. Chi $\mathrm{CH}$, Cho SJ. Improvement of bioactivity of soybean meal by solid-state fermentation with Bacillus amyloliquefaciens versus Lactobacillus spp. and Saccharomyces cerevisiae. LWT-Food Sci Technol. 2016;68:619-25. https://doi.org/10.1016/j.lwt.2015.12.002 